\title{
Insular Volume Reduction in Patients with Social Anxiety Disorder
}

\author{
Akiko Kawaguchi' , Kiyotaka Nemoto ${ }^{2 *}$, Shutaro Nakaaki ${ }^{3}$, Takatsune Kawaguchi ${ }^{4}$, \\ Hirohito Kan ${ }^{5}$, Nobuyuki Arai ${ }^{5}$, Nao Shiraishi ${ }^{1}$, Nobuhiko Hashimoto ${ }^{1}$ and Tatsuo Akechi ${ }^{1}$ \\ 'Department of Psychiatry and Cognitive-Behavioral Medicine, Graduate School of Medical Sciences, Nagoya City \\ University, Nagoya, Japan, ${ }^{2}$ Department of Psychiatry, Faculty of Medicine, University of Tsukuba, Ibaraki, Japan, \\ ${ }^{3}$ Department of Psychiatry, Keio University, Tokyo, Japan, ${ }^{4}$ Department of Radiology, Toyota-Kai Medical Corporation, Kariya \\ Toyota General Hospital, Kariya, Japan, ${ }^{5}$ Department of Radiology, Nagoya City University Hospital, Nagoya, Japan
}

Despite the fact that social anxiety disorder (SAD) is highly prevalent, there have been only a few structural imaging studies. Moreover, most of them reported about a volume reduction in amygdale, which plays a key role in the neural function of SAD. Insula is another region of interest. Its hyperactivity in regard to processing negative emotional information or interoceptive awareness has been detected in patients with SAD. Referring to these studies, we hypothesized that insular volumes might reduce in patients with SAD and made a comparison of insular volumes between 13 patients with SAD and

OPEN ACCESS

Edited by:

Takahiro A. Kato,

Kyushu University, Japan

Reviewed by:

Rajeev Krishnadas,

NHS Greater Glasgow and Clyde and University of Glasgow, UK Naoki Hashimoto,

Hokkaido University, Japan

${ }^{*}$ Correspondence: Kiyotaka Nemoto kiyotaka@nemotos.net

Specialty section: This article was submitted to Neuroimaging and Stimulation, a section of the journal Frontiers in Psychiatry

Received: 05 October 2015 Accepted: 07 January 2016 Published: 21 January 2016

Citation: Kawaguchi A, Nemoto K, Nakaaki S, Kawaguchi T, Kan H, Arai N, Shiraishi N, Hashimoto $N$ and Akechi T (2016) Insular Volume Reduction in Patients with Social Anxiety Disorder.

Front. Psychiatry 7:3. doi: 10.3389/fpsyt.2016.00003
18 healthy controls with matched age and gender using voxel-based morphometry. As a result, we found a significant volume reduction in insula in the SAD group. Our results suggest that the patients with SAD might have an insular volume reduction apart from amygdala. Since insula plays a critical role in the pathology of SAD, more attention should be paid not only to functional study but also morphometrical study of insula.

Keywords: social anxiety disorder, magnetic resonance imaging, insula, voxel-based morphometry

\section{INTRODUCTION}

Social anxiety disorder (SAD), also known as social phobia, is defined by excessive fear and avoidance of social situations. According to Clark and Wells (1), patients with SAD tend to have negative social cognitions in the situation in which they fear and these cognitions are enhanced by misperception of somatic symptoms.

Social anxiety disorder is one of the common psychiatric disorders (2) with a lifetime prevalence of $12 \%$ (3). Despite the fact that SAD is highly prevalent, there have been fewer structure MRI studies on SAD than mood disorders or other anxiety disorders. The first study was reported in 1994 by Potts et al. (4), which concluded that there was no statistically significant volume difference between 22 patients with SAD and controls by voxel-based morphometry (VBM) analyses. More than 15 years later, Irle et al. examined hippocampal/amygdala volumes of patients with SAD with region-ofinterest (ROI) method and revealed hippocampal/amygdala volume reduction in patients with SAD (5). They also reported a negative correlation between a right hippocampal volume and Liebowitz Social Anxiety Scale (LSAS) and between a right amygdala volume and State-Trait Anxiety Inventory (STAI). Liao et al. conducted a study which integrated diffusion tensor imaging, resting-state functional magnetic resonance imaging (fMRI), and VBM (6). The study revealed a volume reduction in inferior temporal gyrus and parahippocampal/hippocampal gyrus and a volume increase in right medial prefrontal cortex (MPFC) in patients with SAD. Meng et al. also reported a volume reduction in bilateral thalami, right amygdala, and right precuneus, a negative correlation of a right amygdala 
volume with the duration of disease, and a positive correlation with the age of onset (7). They also suggested that the volume change can occur in the aberrant functional region. Although a few studies $(8,9)$ reported a volume increase in these regions, most of the studies mentioned a volume reduction in hippocam$\mathrm{pal} / \mathrm{amygdala}$, which play key roles in the neural function of SAD according to the fMRI studies (10). Moreover, posttraumatic disorders $(10,11)$ and panic disorders $(12-15)$, which are supposed to have a shared anxiety-related neural network with SAD, also present both hyperactivity and a volume reduction in amygdala. According to these perspectives, the same phenomenon could occur in patients with SAD. In other words, hyperactivity might accompany a volume reduction as Meg et al. mentioned.

In addition to hippocampus or amygdala, insula is another ROI. Insula is located in the central region of the cerebral, and it has connection with many cortical areas including anterior cingulate cortex (ACC), PFC, somatosensory areas, and limbic areas, such as amygdala (16). It has various roles, for instance, processing visceral sensory, visceral motor, vestibular, motor information (17), romantic love (18), and maternal love (19), and dealing with strong emotions, such as social pain (20) or a variety of negative emotions (21). Among those various roles, perceiving strong emotions and processing introspective awareness are major roles of insula (22). Previous fMRI studies in patients with SAD have detected hyperactivity of insula, and this has been related to the excessive processing of negative feeling, such as disgust or anger (10, 23-29). In those situations, insula is coactivated with amygdala and evaluates the emotional stimuli (30).

Another major role of insula is the interoception, which is defined as the awareness of the internal bodily state. When we feel heartbeat, or sense a touch or pain on skin, insula conveys these perceptions. This internal awareness is important to realize the bodily state or the image of the self (self-awareness) (31). Insula is involved in the excessive processing of the interoceptive state in patients with SAD, and this information is sent to the ACC, and ACC generates an altered prediction signal $(32,33)$. This signal causes the initiation of action, resulting in the safety behaviors or avoidance.

The network between insula and dorsal ACC has been known as "saliency network" $(34,35)$. This network is related to bottomup detection of salient events, switching between other large-scale networks to facilitate access to attention and working memory resources when a salient event is detected, modulating autonomic reactivity to salient stimuli, and strong functional coupling with the ACC that facilitates rapid access to the motor system (34). According to the Clark and Wells model (1), which is the most well-known psychological model of SAD, patients with SAD tend to have negative social cognitions when they face the social situations and this leads to enhance the several somatic symptoms, such as palpitation, blush, and shivering. Then, they overestimate these symptoms as threatening matters and this cause much more negative social cognition (e.g., surrounding people might notice their somatic symptoms and people might think the patient is unworthiness). This can be viewed as inappropriate activation of saliency network. Indeed, there is a report which demonstrate decreased functional coupling of the insula within the saliency network in patients with SAD (36).
Considering this, insula could play such an important role in $\mathrm{SAD}$. Therefore, we hypothesized that the insular volume is likely to reduce as well as the amygdala and hippocampus. A recent study reported a reduction of cortical thickness of insula in SAD by the ROI analyses (37), which is in line with our hypothesis. We investigated whether insular volume would reduce in patients with SAD in comparison with healthy individuals using VBM.

\section{MATERIALS AND METHODS}

\section{Subjects}

All the patients were enrolled from the department of psychiatry at Nagoya City University Hospital. They met the criteria for generalized subtype SAD as the primary disorder, which were determined with the structured clinical interview for DSM-IV Axis I disorders (SCID-I). The control (CTL) group consisted of people who did not have concurrent and past Axis I disorders without the family history of psychiatric problems within the second degree relatives and were recruited from the local population. All the subjects were Japanese, right handed, which was confirmed based on the Edinburgh handedness inventory (38). We excluded the participants who had a history of major medical or neurological illness, significant head trauma, or a lifetime history of alcohol or drug dependence. As a result, the subjects recruited for this study consisted of 13 outpatients with SAD (five males and eight females; age range, $20-56$ years; mean \pm SD, $36.2 \pm 11.8$ years) and 18 healthy CTL individuals with similar age and sex (six males and 12 females; age range, 21-53 years; mean \pm SD, $33.8 \pm 9.6$ years). The onset and duration of SAD $($ mean $\pm \mathrm{SD})$ were $13.0 \pm 10.3$ years old and $23.4 \pm 14.4$ years, respectively. Concurrent and past comorbidities of the patients were as follows: four patients had concurrent comorbid major depressive disorders, two had past major depressive disorders, one had a concurrent panic disorder, one had a past panic disorder, one had a concurrent bulimia nervosa, and one had a past bulimia nervosa and past adjustment disorder. Although there were some patients with concurrent Axis I disorders, SAD was their primary disorder, and those comorbid symptoms were under control to the extent that they managed to go through MRI scanning. More detailed characteristics of the participants are summarized in Table 1. The Ethics Committee of Nagoya City University Graduate School of Medical Sciences approved this study. After being explained about the study, all the subjects provided written informed consent.

\section{Psychiatric Assessment}

The degrees of social anxiety were assessed by the LSAS, Social Interaction Anxiety Scale/Social Phobia Scale (SIAS/SPS), Brief Version of the Fear of Negative Evaluation Scale (BFNE), Hamilton Rating Scale for Depression (HRSD), and Japanese version of National Adult Reading Test (JART).

\section{Liebowitz Social Anxiety Scale}

Liebowitz Social Anxiety Scale is the most popular rater-administered assessment tool for the social anxiety with 24 performance or social interaction situations (39). It provides separate scores for 
TABLE 1 | Demographic data of subjects.

\begin{tabular}{|c|c|c|c|c|}
\hline & & SAD group & CTL group & $p$ \\
\hline \multicolumn{2}{|l|}{ Number } & 13 & 18 & \\
\hline \multirow[t]{2}{*}{ Gender } & Male & 5 & 6 & 1 \\
\hline & Female & 8 & 12 & \\
\hline \multicolumn{2}{|c|}{ Age mean (SD) } & $36.2(11.8)$ & $33.8(9.6)$ & 0.5 \\
\hline \multicolumn{2}{|l|}{ IQ mean (SD) } & $106.2(10.9)$ & $109.3(5.8)$ & 0.3 \\
\hline \multicolumn{2}{|c|}{ LSAS mean (SD) } & $81.6(14.3)$ & $25.6(14.2)$ & $<0.001^{\star \star}$ \\
\hline \multicolumn{2}{|c|}{ BFNE mean (SD) } & $35.3(6.4)$ & $18.6(7.8)$ & $<0.001^{\text {** }}$ \\
\hline \multicolumn{2}{|c|}{ SPS mean (SD) } & $37.2(14.2)$ & $7.1(4.6)$ & $<0.001^{\text {** }}$ \\
\hline \multicolumn{2}{|c|}{ SIAS mean (SD) } & $52.9(10.1)$ & $24.4(10.2)$ & $<0.001^{\star \star}$ \\
\hline \multicolumn{2}{|c|}{ HRSD mean (SD) } & $8.8(5.2)$ & $0.8(1.6)$ & $<0.001^{\star \star}$ \\
\hline \multicolumn{2}{|c|}{ Age of onset mean (SD) } & $13.0(10.3)$ & NA & \\
\hline \multicolumn{2}{|c|}{ Duration of SAD years mean (SD) } & $23.3(14.4)$ & NA & \\
\hline \multicolumn{2}{|c|}{ Antidepressants DDD mean (SD) } & $1.1(0.8)$ & NA & \\
\hline \multicolumn{2}{|c|}{ Antianxiety agents DDD mean (SD) } & $0.7(0.8)$ & NA & \\
\hline \multicolumn{2}{|c|}{ Hypnotics DDD mean (SD) } & $0.4(0.9)$ & NA & \\
\hline \multicolumn{2}{|c|}{ Antipsychotics DDD mean (SD) } & $0.1(0.2)$ & NA & \\
\hline \multicolumn{2}{|c|}{ Cognitive behavioral therapy (\%) } & $9(69.2)$ & NA & \\
\hline \multirow[t]{3}{*}{$\begin{array}{l}\text { Current } \\
\text { comorbidity }\end{array}$} & $\begin{array}{l}\text { Major depressive } \\
\text { disorder (\%) }\end{array}$ & $4(30.8)$ & NA & \\
\hline & Panic disorder (\%) & $1(7.7)$ & NA & \\
\hline & Bulimia nervosa (\%) & $1(7.7)$ & NA & \\
\hline \multirow[t]{4}{*}{$\begin{array}{l}\text { Past } \\
\text { comorbidity }\end{array}$} & $\begin{array}{l}\text { Major depressive } \\
\text { disorder (\%) }\end{array}$ & $2(15.4)$ & NA & \\
\hline & Panic disorder (\%) & $1(7.7)$ & NA & \\
\hline & Bulimia nervosa (\%) & $1(7.7)$ & NA & \\
\hline & $\begin{array}{l}\text { Adjustment } \\
\text { disorder (\%) }\end{array}$ & $1(7.7)$ & NA & \\
\hline \multicolumn{2}{|c|}{ Gray matter volume mean (SD) } & $533.5(50.9)$ & $577.3(72.6)$ & 0.1 \\
\hline \multicolumn{2}{|c|}{ White matter volume mean (SD) } & $640.0(52.3)$ & $684.4(88.1)$ & 0.07 \\
\hline
\end{tabular}

$S A D$, social anxiety disorder; CTL, control group; IQ, Intelligence Quotient; LSAS, Liebowitz Social Anxiety Scale; BFNE, Brief Version of the Fear of Negative Evaluation Scale; SPS, Social Phobia Scale; SIAS, Social Interaction Anxiety Scale; HRSD, Hamilton Rating Scale for Depression; DDD, defined daily dose; NA, not applicable. ${ }^{* *} p<0.001$.

fear (0-3 indicate none, mild, moderate, and severe, respectively) and avoidance ( $0-3$ indicate never, occasionally, often, and usually, respectively) of the situation. High score indicates severe symptoms. Reliability and validity have been demonstrated sufficiently for both original and Japanese versions (40).

\section{Social Interaction Anxiety Scale/Social Phobia Scale}

Social Interaction Anxiety Scale/Social Phobia Scale are 20-item self-report questionnaires with ratings on a 4-point scale from 0 (not at all characteristic or true of me) to 4 (extremely characteristic or true of me) (41). Its total scores ranges from 0 to 80 , and high score indicates severe symptoms. The SPS measures the fear of being observed, whereas the SIAS provides a measure of fear of social interaction. Sufficient reliability and validity have been demonstrated for both original and Japanese versions (42).
Brief Version of the Fear of Negative Evaluation Scale Brief Version of the Fear of Negative Evaluation Scale (BFNE) assesses the fear of negative evaluation in social situations (43). The BFNE consists of 12 questions with a 1 (not at all characteristic of me) to 5 (extremely characteristic of me) point. Sufficient reliability and validity of the Japanese version have been reported in FNE (44).

\section{Hamilton Rating Scale for Depression}

Hamilton Rating Scale for Depression is the most frequently used clinician-administered ratings for depression (45). It contains 17 items and each item is scored on a 3 - or 5-point scale, depending on the item. The total score estimates the severity of the depressive symptom. Reliability and validity have been demonstrated even in Japanese version (46).

\section{Japanese Version of National Adult Reading Test}

Japanese version of National Adult Reading Test was established according to the National Adult Reading Test (NART) (47). It contains 50 words reading kanji and estimates Intelligence Quotient (IQ). Reliability and validity have been demonstrated sufficiently.

All the rater assessments from psychiatric perspectives were carried out by a board-certified psychiatrist (Akiko Kawaguchi).

\section{MRI Image Acquisitions}

Three-dimensional (3D) magnetization-prepared rapid gradient echo (MP-RAGE) images were acquired using a 3-T MR scanner (Magnetom Skyra, Siemens Medical Solutions, Erlangen, Germany) and a 32-channel head array coil. The parameters were as follows: repetition time, $7.3 \mathrm{~ms}$; echo time (TE), $2.43 \mathrm{~ms}$; flip angle, $9^{\circ}$; inversion time, $900 \mathrm{~ms}$; matrix size, $256 \times 256$; slice thickness, $1 \mathrm{~mm}$; and the number of sagittal images, 176 in total. A board-certified neuroradiologist (Takatsune Kawaguchi) reviewed all the scanned images and found no major abnormalities in any of the subjects.

\section{Demographic Data Analysis}

Behavioral data was analyzed using SPSS version 19.0 software (IBM Corp. Armonk, NY, USA). Psychiatric measurements or demographic data were analyzed with unpaired $t$-tests for continuous variables and $\chi^{2}$ square test for categorical variables to compare the SAD and CTL groups. Results were considered statistically significant at $p<0.05$.

\section{Imaging Data Analysis}

All images were preprocessed and analyzed using Statistical Parametric Mapping 8 (SPM8 ${ }^{1}$ ) running on Matlab R2013b (Mathworks Inv., Sherborn, MA, USA). VBM was performed using the VBM8 toolbox. ${ }^{2}$ Each MPRAGE image was segmented into gray matter (GM), white matter (WM), and cerebrospinal fluid (CSF). Subsequently, the segmented GM images were spatially normalized using the diffeomorphic anatomical registration

\footnotetext{
${ }^{1}$ http://www.fil.ion.ucl.ac.uk/spm/
}

${ }^{2} \mathrm{http}: / / \mathrm{dbm}$. neuro.uni-jena.de/vbm 
through exponentiated lie algebra (DARTEL) algorithm. The voxel values were modulated by Jacobian determinants for the non-linear components ("non-linear only" option in VBM8 toolbox), and smoothed with an 8 -mm full width at half maximum (FWHM) Gaussian kernel.

After preprocessing, two sample $t$-tests were performed between the SAD and the CTL groups to detect regional differences in GM images. We employed a small volume correction (SVC) within bilateral insulae based on our hypothesis. For the SVC analysis, a family-wise error (FWE)-corrected voxel level threshold of $p<0.05$ was applied within bilateral insular ROIs to account for multiple comparisons of the results. ROIs of bilateral insulae were made using automated anatomical labeling (AAL) atlas included in WFU PickAtlas ver. 3.0.4 (48). Voxel coordinates were given in Montreal Neurological Institute (MNI) space and displayed as such.

\section{RESULTS}

\section{Demographic Data}

Demographic and diagnostic characteristics of the participants were summarized in Table 1. Age, gender, and estimated IQ were not significantly different between the groups. On the other hand, SAD group showed significant higher scores than CTL in LSAS (SAD $81.6 \pm 14.3$; CTL $25.6 \pm 14.2$ ), SIAS/SPS (SAD $52.9 \pm 10.1 / 37.2 \pm 14.2$; CTL $24.2 \pm 10.2 / 7.1 \pm 4.6)$, and BFNE $($ SAD $35.3 \pm 6.4 ;$ CTL $18.6 \pm 7.8)$.

\section{MRI Results}

The whole-brain two sample $t$-tests showed no statistically significant differences between the SAD group and the CTL after multiple comparisons. Confining the ROIs to bilateral insulae, we found that left anterior and right posterior insular volumes significantly reduced in the SAD group as compared to the CTL (Figure 1; Table 2). We did not find significant correlation between insular volumes and LSAS, SIAS/SPS, or BFNE. We also investigated the difference in hippocampus and amygdala according to the previous reports, which resulted in no significant differences between the groups.

\section{DISCUSSION}

\section{Main Findings}

As we had expected, we found a statistically significant group difference in the insular volume between the SAD and the CTL groups. Bilateral insular volume was reduced in patients with $\mathrm{SAD}$. There has been only one study investigating the relationship between insula and SAD, which reported cortical thinning of right insula (37). Our results reinforced it. Syal et al. implicated the altered interoception and aberrant insular-ACC connection in concern with interoception as reasons for the reduction.

Social anxiety disorder has been explained with cognitive behavioral model (1). According to the model, SAD patients tend to have negative social cognitions and these cognitions are enhanced by safety behaviors or somatic symptoms. Since this model focuses on negative cognition or feelings, most of the previous neuroimaging studies on SAD investigated mainly hippocampus or amygdala (10). Volume reduction or hyperactivation

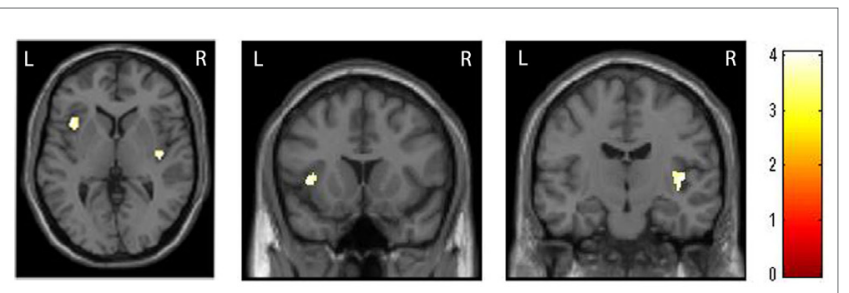

FIGURE 1 | Comparison of insular volumes between patients with social anxiety disorder (SAD) and the control (CTL) group. Reduced bilateral insular volumes occurred in the SAD group as compared to the CTL group. For the statistical analysis, a family-wise error (FWE)-corrected voxel level threshold of $p<0.05$ was applied within bilateral insular regions of interest to account for multiple comparisons of the results.

TABLE 2 | Comparison of insular volumes between patients with SAD and control.

\begin{tabular}{|c|c|c|c|c|c|c|}
\hline \multirow[t]{2}{*}{ Structure } & \multicolumn{3}{|c|}{ MNI coordinates } & \multirow[t]{2}{*}{$T$} & \multirow[t]{2}{*}{$p$} & \multirow[t]{2}{*}{ Cluster size } \\
\hline & $x$ & $Y$ & $z$ & & & \\
\hline Right insula & 42 & -13 & 3 & 4.02 & 0.032 & 157 \\
\hline Left insula & -39 & 15 & 3 & 4.07 & 0.029 & 145 \\
\hline
\end{tabular}

MNI, Montreal Neurological Institute.

of these regions are also observed in other anxiety disorders, such as panic disorder (13-15).

In addition to the Clark and Wells model, misinteroception has been attracting attention. From the misinteroception point of view, SAD patients might recognize their somatic symptoms, such as blushing or trembling, as hazardous alarm to self, which reinforce their negative cognition. This aberrant interoception might be caused by the hyperactivity of insula because interoception is one of the major roles of the insula, and it links the bodily state and affective or cognitive processes (32). Indeed, anxiolytics are known to modulate those insular activations via GABA neuron and reduce the anxiety (49). Menon and Uddin pointed that hyperactivity of insula might be related to the enhanced detection of saliency (34).

Insula, especially anterior insula, has also known to be a center for saliency network. Saliency network comprises mainly of anterior insula and ACC. Anterior insula works as a hub, which integrates the external physiological sensation and sends signals to ACC in order to adjust the behavior (34).

Anterior insula evaluates the meaning of the external stimuli, and ACC generates the signals to PFC for controlling the behavior and cognition. Klumpp et al. detected the altered insula-ACC connection in SAD using fMRI connectivity analysis (26). They suggested that the less connectivity of insular-ACC in patients with SAD compared to the healthy comparisons and insufficient cognitive control of ACC and PFC affected the threat processing.

We observed volume reduction in not only anterior insula but also posterior insula. Posterior insular has reciprocal connections with higher order visual areas, auditory processing areas, somatosensory areas as well as anterior insula, and its function is specialized for directly experienced, multimodal sensory processing, particularly somatosensory processing (16). Nagai et al. also 
points that dysfunction of the posterior insular cortex might lead to visual-somatosensory imbalance, resulting in depersonalization or agoraphobia (17). Taken together, hyperactivity and dysfunction of saliency network and posterior insula might contribute the pathophysiology of SAD as well as hippocampus/amygdala regions.

As mentioned above, hyperactivity of insula is crucial in the neural pathology of SAD, and Meng et al. suggested that a volume change could occur in the aberrant functional region (7). Our results were in line with this idea. There are some functional and structure studies, which show both hyperactivity and a volume reduction in SAD as well as in other anxiety disorders (e.g., similar insular hyperactivity and volume reduction have found in panic disorder, which was also applied the cognitive behavioral model) (13-15). Furthermore, we suggest that in future studies, the effect of pharmacological or psychological treatment, or characteristics of patients on an insular volume should be investigated in large samples. Since treatment reduces the hyperactivity of insula, we expect that accompanied volume reduction might be recovered.

Though previous studies report volume reduction in hippocampus or amygdala, we did not find significant volume reduction in our sample. One of the reasons might be due to the small sample size. Indeed, if we lower the statistical threshold, group comparisons show volume reduction in the amygdala/ hippocampal regions of SAD patients compared to controls ( $p<0.005$, uncorrected for hippocampus and $p<0.05$ uncorrected for amygdala). Taking this into consideration, we suggest that both amygdala/hippocampus and insula contribute to the underlying mechanism of SAD.

In this study, we employed VBM to investigate the volume reduction in $\mathrm{SAD}$. A previous study reported a reduction of cortical thickness of insula in SAD (37). Hutton et al. compared cortical thickness and VBM and suggested that these should be considered as complementary approaches because VBM is additionally sensitive to local surface area and cortical folding as well as cortical thickness (50). Considering this, our results using VBM reinforce the previous report in that insula is involved in the pathophysiology of SAD.

\section{Limitations}

The present study had several limitations. First, some of the participants with SAD had comorbid mood, anxiety, and eating disorders. However, their primary diagnosis was SAD and, as summarized in Table 1, their HRSD scores were in a low level. Second, the effect of the antidepressant and benzodiazepine medications of the patients might have interfered with the outcome. However, there is a large study which shows that the medication does not have much effect on volumetric results among the patients with

\section{REFERENCES}

1. Clark D, Wells A. A cognitive model of social phobia. In: Heimberg RG, Liebowitz M, Hope DA, Schneier FR, editors. Social Phobia: Diagnosis, Assessment and Treatment. New York: Guilford Press (1995). p. 69-93.

2. Liebowitz MR, Heimberg RG, Fresco DM, Travers J, Stein MB. Social phobia or social anxiety disorder: what's in a name? Arch Gen Psychiatry (2000) 57(2):191-2. doi:10.1001/archpsyc.57.2.191-a

3. Kessler RC, Berglund P, Demler O, Jin R, Merikangas KR, Walters EE. Lifetime prevalence and age-of-onset distributions of DSM-IV disorders in the national same severity (51). Furthermore, our study was based on a small sample size, and this might have been the reason for us not to have been able to find a significant group difference in the whole brain analyses. Our result needs to be replicated in a future study with a larger sample.

Although there are several limitations, we suggest the possibility that insula, which plays a critical role in the pathology of SAD, has a volume reduction as well as in amygdala. Considering the importance of the functional role of insula, we should pay more attention to morphometrical study of insula.

\section{AUTHOR CONTRIBUTIONS}

AK designed and managed the study. Image analyses were carried out by AK and TK. KN and SN supervised the image analyses. TK performed the quality control of MRI. HK and NA contributed to MRI data acquisition as radiology technicians. NS and NH managed the patient enrollment. AK carried out statistical analyses and drafted the manuscript under the supervision of $\mathrm{KN}, \mathrm{SN}$, and TA. All authors read and approved the final manuscript.

\section{ACKNOWLEDGMENTS}

We would like to express our sincere appreciation to all the participants. We also would like to thank the radiology technicians of Nagoya City University Hospital (Harumasa Kasai Ph.D., Mr. Hiroshi Kunitomo, Mr. Kiyotaka Mori, Mr. Naohisa Harada, Mr. Yuta Nishiwaki, Mr. Katsuya Kato, and Mr. Takamine Ezaka) and psychiatrists and secretaries of Department of Psychiatry and Cognitive-Behavioral Medicine, Nagoya City University Graduate School of Medical Sciences (particularly Koji Sugano M.D., Ph.D.; Takahiro Yamada M.D., Ph.D.; Masaki Kondo M.D., Ph.D.; Sei Ogawa M.D., Ph.D.; Masako Suzuki M.D., Ph.D.; Tadashi Funayama M.D., Ph.D.; Masahiro Nishioka M.D.; Yuri Kosaki M.D.; Ms. Kaori Kobori; Ms. Kozue Maki; Ms. Ikuyo Sakakima; and Ms. Kyoko Yanagi) for their support. This study was supported by the Department of Psychiatry and Cognitive-Behavioral Medicine, Nagoya City University Graduate School of Medical Sciences, and also by a Grant-in-Aid for Scientific Research on Innovative Areas (Comprehensive Brain Science Network) from the Ministry of Education, Science, Sports and Culture of Japan.

\section{FUNDING}

This study was supported by a Grant-in-Aid for young scientists (25780421) from the Ministry of Education, Science, Sports and Culture of Japan.

comorbidity survey replication. Arch Gen Psychiatry (2005) 62(6):593-602 doi:10.1001/archpsyc.62.6.617

4. Potts NL, Davidson JR, Krishnan KR, Doraiswamy PM. Magnetic resonance imaging in social phobia. Psychiatry Res (1994) 52(1):35-42. doi:10.1016/0165-1781(94)90118-X

5. Irle E, Ruhleder M, Lange C, Seidler-Brandler U, Salzer S, Dechent P, et al. Reduced amygdalar and hippocampal size in adults with generalized social phobia. J Psychiatry Neurosci (2010) 35(2):126-31. doi:10.1503/jpn.090041

6. Liao W, Xu Q, Mantini D, Ding J, Machado-de-Sousa JP, Hallak JE, et al. Altered gray matter morphometry and resting-state functional and 
structural connectivity in social anxiety disorder. Brain Res (2011) 1388:16777. doi:10.1016/j.brainres.2011.03.018

7. Meng Y, Lui S, Qiu C, Qiu L, Lama S, Huang X, et al. Neuroanatomical deficits in drug-naive adult patients with generalized social anxiety disorder: a voxel-based morphometry study. Psychiatry Res (2013) 214(1):9-15. doi:10.1016/j.pscychresns.2013.06.002

8. Talati A, Pantazatos SP, Schneier FR, Weissman MM, Hirsch J. Gray matter abnormalities in social anxiety disorder: primary, replication, and specificity studies. Biol Psychiatry (2013) 73(1):75-84. doi:10.1016/j. biopsych.2012.05.022

9. Machado-de-Sousa JP, Osorio Fde L, Jackowski AP, Bressan RA, Chagas MH, Torro-Alves $\mathrm{N}$, et al. Increased amygdalar and hippocampal volumes in young adults with social anxiety. PLoS One (2014) 9(2):e88523. doi:10.1371/journal. pone. 0088523

10. Etkin A, Wager TD. Functional neuroimaging of anxiety: a meta-analysis of emotional processing in PTSD, social anxiety disorder, and specific phobia. Am J Psychiatry (2007) 164(10):1476-88. doi:10.1176/appi. ajp.2007.07030504

11. Morey RA, Gold AL, LaBar KS, Beall SK, Brown VM, Haswell CC, et al. Amygdala volume changes in posttraumatic stress disorder in a large case-controlled veterans group. Arch Gen Psychiatry (2012) 69(11):1169-78. doi:10.1001/archgenpsychiatry.2012.50

12. Hayano F, Nakamura M, Asami T, Uehara K, Yoshida T, Roppongi $\mathrm{T}$, et al. Smaller amygdala is associated with anxiety in patients with panic disorder. Psychiatry Clin Neurosci (2009) 63(3):266-76. doi:10.1111/j.1440-1819.2009.01960.x

13. Del Casale A, Serata D, Rapinesi C, Kotzalidis GD, Angeletti G, Tatarelli R, et al. Structural neuroimaging in patients with panic disorder: findings and limitations of recent studies. Psychiatr Danub (2013) 25(2):108-14.

14. Wittmann A, Schlagenhauf F, John T, Guhn A, Rehbein H, Siegmund A, et al. A new paradigm (Westphal-Paradigm) to study the neural correlates of panic disorder with agoraphobia. Eur Arch Psychiatry Clin Neurosci (2011) 261(3):185-94. doi:10.1007/s00406-010-0167-1

15. Asami T, Yamasue H, Hayano F, Nakamura M, Uehara K, Otsuka T, et al. Sexually dimorphic gray matter volume reduction in patients with panic disorder. Psychiatry Res (2009) 173(2):128-34. doi:10.1016/j. pscychresns.2008.10.004

16. Wylie KP, Tregellas JR. The role of the insula in schizophrenia. Schizophr Res (2010) 123(2-3):93-104. doi:10.1016/j.schres.2010.08.027

17. Nagai M, Kishi K, Kato S. Insular cortex and neuropsychiatric disorders: a review of recent literature. Eur Psychiatry (2007) 22(6):387-94. doi:10.1016/j. eurpsy.2007.02.006

18. Bartels A, Zeki S. The neural correlates of maternal and romantic love. Neuroimage (2004) 21(3):1155-66. doi:10.1016/j.neuroimage.2003.11.003

19. Leibenluft E, Gobbini MI, Harrison T, Haxby JV. Mothers' neural activation in response to pictures of their children and other children. Biol Psychiatry (2004) 56(4):225-32. doi:10.1016/j.biopsych.2004.05.017

20. Eisenberger NI, Lieberman MD, Williams KD. Does rejection hurt? An fMRI study of social exclusion. Science (2003) 302(5643):290-2. doi:10.1126/ science. 1089134

21. Phan KL, Wager T, Taylor SF, Liberzon I. Functional neuroanatomy of emotion: a meta-analysis of emotion activation studies in PET and fMRI. Neuroimage (2002) 16(2):331-48. doi:10.1006/nimg.2002.1087

22. Critchley HD, Wiens S, Rotshtein P, Ohman A, Dolan RJ. Neural systems supporting interoceptive awareness. Nat Neurosci (2004) 7(2):189-95. doi: $10.1038 / \mathrm{nn} 1176$

23. Amir N, Klumpp H, Elias J, Bedwell JS, Yanasak N, Miller LS. Increased activation of the anterior cingulate cortex during processing of disgust faces in individuals with social phobia. Biol Psychiatry (2005) 57(9):975-81. doi:10.1016/j.biopsych.2005.01.044

24. Ball TM, Sullivan S, Flagan T, Hitchcock CA, Simmons A, Paulus MP, et al. Selective effects of social anxiety, anxiety sensitivity, and negative affectivity on the neural bases of emotional face processing. Neuroimage (2012) 59(2):1879-87. doi:10.1016/j.neuroimage.2011.08.074

25. Evans KC, Wright CI, Wedig MM, Gold AL, Pollack MH, Rauch SL. A functional MRI study of amygdala responses to angry schematic faces in social anxiety disorder. Depress Anxiety (2008) 25(6):496-505. doi:10.1002/ da. 20347
26. Klumpp H, Angstadt M, Phan KL. Insula reactivity and connectivity to anterior cingulate cortex when processing threat in generalized social anxiety disorder. Biol Psychol (2012) 89(1):273-6. doi:10.1016/j.biopsycho.2011.10.010

27. Schmidt S, Mohr A, Miltner WH, Straube T. Task-dependent neural correlates of the processing of verbal threat-related stimuli in social phobia. Biol Psychol (2010) 84(2):304-12. doi:10.1016/j.biopsycho.2010.03.005

28. Shah SG, Klumpp H, Angstadt M, Nathan PJ, Phan KL. Amygdala and insula response to emotional images in patients with generalized social anxiety disorder. J Psychiatry Neurosci (2009) 34(4):296-302.

29. Straube T, Kolassa IT, Glauer M, Mentzel HJ, Miltner WH. Effect of task conditions on brain responses to threatening faces in social phobics: an event-related functional magnetic resonance imaging study. Biol Psychiatry (2004) 56(12):921-30. doi:10.1016/j.biopsych.2004.09.024

30. Lovero KL, Simmons AN, Aron JL, Paulus MP. Anterior insular cortex anticipates impending stimulus significance. Neuroimage (2009) 45(3):976-83. doi:10.1016/j.neuroimage.2008.12.070

31. Devue C, Collette F, Balteau E, Degueldre C, Luxen A, Maquet P, et al. Here I am: the cortical correlates of visual self-recognition. Brain Res (2007) 1143:169-82. doi:10.1016/j.brainres.2007.01.055

32. Paulus MP, Stein MB. An insular view of anxiety. Biol Psychiatry (2006) 60(4):383-7. doi:10.1016/j.biopsych.2006.03.042

33. Critchley HD, Harrison NA. Visceral influences on brain and behavior. Neuron (2013) 77(4):624-38. doi:10.1016/j.neuron.2013.02.008

34. Menon V, Uddin LQ. Saliency, switching, attention and control: a network model of insula function. Brain Struct Funct (2010) 214(5-6):655-67. doi:10.1007/s00429-010-0262-0

35. Seeley WW, Menon V, Schatzberg AF, Keller J, Glover GH, Kenna H, et al. Dissociable intrinsic connectivity networks for salience processing and executive control. J Neurosci (2007) 27(9):2349-56. doi:10.1523/ JNEUROSCI.5587-06.2007

36. Liao W, Chen H, Feng Y, Mantini D, Gentili C, Pan Z, et al. Selective aberrant functional connectivity of resting state networks in social anxiety disorder. Neuroimage (2010) 52(4):1549-58. doi:10.1016/j.neuroimage.2010.05.010

37. Syal S, Hattingh CJ, Fouche JP, Spottiswoode B, Carey PD, Lochner C, et al. Grey matter abnormalities in social anxiety disorder: a pilot study. Metab Brain Dis (2012) 27(3):299-309. doi:10.1007/s11011-012-9299-5

38. Oldfield RC. The assessment and analysis of handedness: the Edinburgh inventory.Neuropsychologia(1971) 9(1):97-113.doi:10.1016/0028-3932(71)90067-4

39. Liebowitz MR. Social phobia. Mod Probl Pharmacopsychiatry (1987) 22:141-73. doi:10.1159/000414022

40. Asakura S, Inoue S, Sasaki F, Sasaki YK, Kitagawa N, Inoue T, et al. Reliability and validity of the Japanese version of the Liebowitz social anxiety scale. Clin Psychiatry (2002) 44:1077-84.

41. Mattick RP, Clarke JC. Development and validation of measures of social phobia scrutiny fear and social interaction anxiety. Behav Res Ther (1998) 36(4):455-70. doi:10.1016/S0005-7967(97)10031-6

42. Kanai Y, Sasakawa S, Chen J, Suzuki S, Shimada H, Sakano Y. Development and validation of the Japanese version of social phobia scale and social interaction anxiety scale. Jpn J Psychosom Med (2004) 44:841-50.

43. Leary MR. A brief version of the fear of negative evaluation scale. Pers Soc Psychol Bull (1983) 9:371-5. doi:10.1177/0146167283093007

44. Ishikawa RSK, Fukui I. Standardization of Japanese version of FNE and SADS. Jap J Behav Ther (1992) 18:10-7.

45. Hamilton M. A rating scale for depression. J Neurol Neurosurg Psychiatry (1960) 23:56-62. doi:10.1136/jnnp.23.1.56

46. Furukawa TA, Akechi T, Azuma H, Okuyama T, Higuchi T. Evidencebased guidelines for interpretation of the Hamilton Rating Scale for Depression. J Clin Psychopharmacol (2007) 27(5):531-4. doi:10.1097/ JCP.0b013e31814f30b1

47. Matsuoka K, Uno M, Kasai K, Koyama K, Kim Y. Estimation of premorbid IQ in individuals with Alzheimer's disease using Japanese ideographic script (Kanji) compound words: Japanese version of National Adult Reading Test. Psychiatry Clin Neurosci (2006) 60(3):332-9. doi:10.1111/j.1440-1819.2006.01510.x

48. Maldjian JA, Laurienti PJ, Kraft RA, Burdette JH. An automated method for neuroanatomic and cytoarchitectonic atlas-based interrogation of fMRI data sets. Neuroimage (2003) 19(3):1233-9. doi:10.1016/ S1053-8119(03)00169-1 
49. Paulus MP, Feinstein JS, Castillo G, Simmons AN, Stein MB. Dose-dependent decrease of activation in bilateral amygdala and insula by lorazepam during emotion processing. Arch Gen Psychiatry (2005) 62(3):282-8. doi:10.1001/ archpsyc.62.3.282

50. Hutton C, Draganski B, Ashburner J, Weiskopf N. A comparison between voxel-based cortical thickness and voxel-based morphometry in normal aging. Neuroimage (2009) 48(2):371-80. doi:10.1016/j.neuroimage.2009.06.043

51. van Tol MJ, van der Wee NJ, van den Heuvel OA, Nielen MM, Demenescu LR, Aleman A, et al. Regional brain volume in depression and anxiety disorders. Arch Gen Psychiatry (2010) 67(10):1002-11. doi:10.1001/ archgenpsychiatry.2010.121
Conflict of Interest Statement: The authors declare that the research was conducted in the absence of any commercial or financial relationships that could be construed as a potential conflict of interest.

Copyright $\odot 2016$ Kawaguchi, Nemoto, Nakaaki, Kawaguchi, Kan, Arai, Shiraishi, Hashimoto and Akechi. This is an open-access article distributed under the terms of the Creative Commons Attribution License (CC BY). The use, distribution or reproduction in other forums is permitted, provided the original author (s) or licensor are credited and that the original publication in this journal is cited, in accordance with accepted academic practice. No use, distribution or reproduction is permitted which does not comply with these terms. 